\title{
PALEOGRAFÍA DE LA BAJA EDAD MEDIA CASTELLANA
}

El tema de la historia de la escritura de la Baja Edad Media en la corona castellano-leonesa plantea, aun a nivel de "status quaestionis», toda una serie de complejas dificultades que se derivan, en principio, de la propia amplitud de su desarrollo, tanto en el espacio como en el tiempo. A ello viene a añadirse la práctica inexistencia de estudios parciales sobre el periodo que nos ocupa ${ }^{\text {, }}$ habiéndose realizado a nivel global síntesis didácticas en manuales que para la escritura libraria suelen remitirse a una breve relación de códices ejecutados en los distintos tipos de escritura, y para la documental no suelen ir más allá de pequeñas indicaciones, como las califica M. Lucas ${ }^{2}$, sobre su morfología, aplicada a nomenclaturas hoy totalmente obsoletas.

No creo necesario hacer hincapié en la amplitud geográfica del área a estudiar. Sí en cambio quiero hacerlo en el cronológico dado que, considerando que el Dr. Núñez Contreras desarrolla su lección exclusivamente sobre el ciclo de la escritura visigótica, creo necesario referirme, antes de entrar de lleno en el ciclo de la escritura gótica, ciclo plenamente bajomedieval, al ciclo de la escritura carolina que, si bien para algunos podría exceder al periodo cronológico que me ha sido encomendado, ha de tener en consideración, ya que en él y para los reinos occidentales de la Península Ibérica, desde su mismo inicio, aparecen los primeros síntomas y signos de evolución que preludian la formación de la escritura gótica.

A la dificultad, ya manifestada, de la amplitud cronológica y geográfica del tema propuesto, viene a sumarse un nuevo problema, tal vez de mayor gravedad y que creo ha influído de manera determinante en la inexistencia de estudios sobre la escritura de los ciclos carolino y gótico: el tema de la nomenclatura.

1 Si exceptuamos el trabajo de M. LUCAS, Características paleográficas de la escritura gótica gallega. Escritorios notariales compostelanos, "Cuadernos de estudios gallegos», XV (1950), pp. 53-86, y el de M. C. ÁlvareZ MÁrQueZ, Escritura latina en la Plena y Baja Edad Media: la llamada "gótica libraria» en España, «Historia. Instituciones. Documentos», 12 (1986), pp.377-410, publicada con posterioridad a la pronunciación de esta lección, la bibliografía queda reducida a los capítulos correspondientes a los manuales al uso en las diferentes épocas.

${ }^{2}$ Cf. M. LUCAS, ob.cit., p. 60. 
Establecidas unas sólidas bases metodológicas de análisis de la escritura por parte de las escuelas franco-belga e italiana, análisis perfectamente aplicable a toda escritura y, por tanto, a las escrituras del periodo que nos ocupa, el historiador de la escritura española choca, al menos en lo que se refiere a las coronas de Castilla y León, con el uso acostumbrado de una terminología totalmente inadecuable a las nuevas tendencias de carácter universalista, encaminadas a resaltar, como destaca $\mathrm{A}$. Petrucci, el fondo común que justifica la adjudicación de una escritura a un periodo y tipo determinado ${ }^{3}$. Términos como minúscula diplomática, letra de privilegios, letra de albalaes, escritura cortesana y procesal, arraigadas en nuestra tradición paleográfica, aparecen vacías de todo sentido para un estudioso no ducho en temas castellanos y oscilan, por otra parte, desde particularismos que las relacionan con un tipo diplomático concreto, hasta la máxima generalización que reserva para denominación de un tipo concreto de escritura, que se desarrolla en un momento determinado como es la Plena Edad Media, una denominación, minúscula diplomática, que etimológicamente es aplicable a cualquier escritura minúscula utilizada en cualquier momento para la redacción de documentos.

Esta dificultad debiera haber quedado resuelta, al menos en cuanto se refiere a la escritura libraria, tras las propuestas realizadas por B. Bischoff, G.I. Lieftinck y G. Battelli en el Primer Coloquio Internacional de Paleografía, celebrado en París en mayo de 1953 y dedicado de manera exclusiva al tema de la terminología paleográfica libraria ${ }^{4}$. Otro tanto pudiera decirse respecto a la escritura documental tras la propuesta enunciada tan sólo dos años después por F. Bartoloni '. Pero la realidad nos lleva a constatar el hecho de que el problema de la terminología paleográfica es aún hoy día un tema abierto y que la dificultad para su correcta resolución es mayor en los países que, como España, Italia y Alemania, se resisten a todo proyecto inmediato de aplicación de una terminología normalizada, dado el peso que en los mismos tiene la terminología tradicional ${ }^{\circ}$.

Ante todo ello, junto al «status quaestionis», más que unas conclusiones, a las que creo imposible acceder en algo tan breve como esta lección, voy a plantear lo que considero son en este momento «desiderata» posibles de realizar. 1984, p.60.

Cf. A. PetrucCI, La descrizione del manoscritto. Storia, problemi, modelli, Roma,

"Vid. Nomenclature des écritures livresques du Xle au XVe siècle, Paris, 1954 ("Colloques Internationaux du CNRS», Sciences humaines, IV).

'La nomenclatura delle scritture documentaire, en «Relazioni del X Congresso Internazionale di Scienze Storiche», I, Firenze, 1955, pp. 434-443.

- Cf. F. GASPARRI, La terminologie des écritures. Rapport présenté à la Table ronde "Nomenclature et Terminologie», en «Paläographie 1981. Colloquium du Comité International de Paleógraphie», München, 1982, pp. 31-37. 
Para el periodo escriturario carolino, en lo que a escritura libraria se refiere, contamos, por una parte, con la visión de conjunto aportada por $\mathbf{A}$. Millares, ciertamente brevísima ', y las láminas seleccionadas y comentadas por A. Canellas en sus Exempla ${ }^{8}$, ambas obras de marcado carácter didáctico.

Quedan, por lo tanto, por establecer aún hechos tan importantes como el «iter» seguido por la escritura carolina libraria en los reinos de Castilla y León, «iter» que, por otra parte, nos facilitaría el establecimiento de posibles vinculaciones entre «scriptoria» castellano-leoneses con «scriptoria» franceses o de las coronas de Aragón, Navarra y Portugal.

Una segunda labor iría encaminada a la identificación de "scriptoria».

Ambas labores se ven dificultadas, por cierto, al no haberse iniciado aún en España la publicación del catálogo de códices datados o datables, obra que serviría de gran ayuda en este empeño.

En lo que hace referencia a la escritura documental castellano-leonesa del ciclo carolino, habría ya, en principio, que partir de una base cierta como es la casi inexistencia de una carolina pura. Su tardía implantación en ambas coronas determina que los tipos utilizados sean fundamentalmente los denominados por J. Stiennon gotizantes y gotizados ${ }^{9}$ y neogóticos o de transición por G. Cencetti ".".

Dentro de la uniformidad básica que supone la práctica de una escritura minúscula sentada, elaborada sobre una misma morfología esencial alfabética, dentro de la escritura documental carolina se pueden apreciar dos tipos diferentes.

El primero lo conforma una carolina común, que interpreta las formas alfabéticas esenciales sin ninguna concesión a la estética del documento y que es la usada por la mayor parte de los rogatarios de documentos particulares y en los cancillerescos no solemnes.

El segundo tipo presenta una escritura carolina de astas alargadas, con los extremos curvados, la llamada minúscula diplomática, que no es sino la interpretación cancilleresca del alfabeto de la carolina común, interpretación que es utilizada en la cancillería real para los documentos de mayor solemnidad y que es asimismo también utilizada en cancillerías eclesiásticas y por rogatarios de documentos privados, fundamentalmente clérigos, que han entrado en contacto con la misma a través de estas cancillerías ".

- Cf. Tratado de Paleografía Española, I, Madrid, 1983, pp. 184-187.

" Exempla scripturarum latinarum in usum scholarum, Pars altera, "Cesaraugustae» MDCCCCLXVI, láms. XXXIV, XXXV, XXXVIII, XLI y XLII.

"Cf. Paleógraphie du Moyen Age, Paris, 1973, pp. 107-110.

10 C.f. Lineamenti di Storia della scrittura latina, Bologna, 1954, p. 22.

$"$ Vid. J. STIENNON, L'écriture diplomatique dans la diocèse de Liège du XIe au milien dı XIlle siècle. Reflet d'une civilisation, Paris, 1960. 
Son, pues, las dos versiones constantemente elaboradas sobre la base de una escritura usual única, la común y la cancilleresca, que en el caso de la carolina castellano-leonesa irán, al avanzar el siglo XII, presentando cada vez mayores rasgos de goticismo, valorables fundamentalmente, aparte de por el despiezado en el trazado de las formas alfabéticas, por el predominio de la $d$ uncial sobre la semiuncial y por la fusión de curvas opuestas.

Sería de gran utilidad, en el campo de la escritura carolina documental castellano-leonesa, la realización de estudios relacionados con la implantación de la misma, implantación muy probablemente relacionada con la presencia en los «scriptoria» monacales de monjes primero franceses y posteriormente ya educados por éstos en el nuevo modelo escriturario ${ }^{12}$. Hallámosnos nuevamente ante la influencia cluniacense, influencia francesa que se extiende a la cancillería real en tiempos de Alfonso VI y que se reforzará, en el caso de este oficio cancilleresco, durante el reinado de Alfonso VII con la llegada de Hugo y Giraldo ${ }^{13}$. Por medio de este estudio podrían, incluso, llegar también a establecerse grados de relación entre diferentes monasterios y caracterizar tipificaciones locales o zonales.

Otro tema pendiente es el estudio de la mayor o menor celeridad en el paso de la carolina gotizante a la gótica y, a través de la carolina gotizada, establecer su diversificación hacia los dos grandes tipos góticos documentales, el sentado o formado y el cursivo.

Y con ello entramos en el riquísimo y complejo ciclo de las escrituras góticas, periodo de una amplia eclosión escrituraria, producto de una serie de coincidencias socioculturales (mundo urbano, cultura laica, florecimiento mercantil) que, partiendo de la unidad gráfica de la escritura carolina, va a cerrarse, si no bajo el signo del particularismo, sí al menos con una extrema variedad de articulaciones locales o funcionales de la escritura ${ }^{14}$.

Por otra parte, si el ciclo carolino basó su quehacer gráfico en una escritura sentada, el ciclo gótico, junto a la utilización de tipos sentados, va a presenciar el nacimiento de una nueva cursiva cuyo preludio se vislumbra en algunas carolinas gotizadas, como anteriormente he indicado, y en cuya base sitúa $\mathrm{A}$. Petrucci como circunstancias culturales condicionantes la difusión del notariado a toda nación europea (no debemos olvidar que en la corona castellanoleonesa el notariado romanista no se institucionalizará hasta el reinado de Alfonso X, mediado el siglo XIII), el uso abundante de la escritura, también a

12 He podido constatar tal circunstancia en la Asturias del siglo XII donde, mientras los monges scriptores del monasterio de San Vicente utilizan la carolina, los notarii rurales continúan utilizando la visigótica. Cf. Más documentos del monasterio de San Vicente anteriores a 1200, "Asturiensia Medievalia», 5 (en prensa).

13 Cf. Millares, ob.cit., I, pp. 168-169.

14 Cf. CenCETTI, ob.cit., p. 246. 
nivel privado, impuesto por el desarrollo mercantil y artesanal, el influjo de las universidades y las producciones literarias, poéticas y narrativas, en lengua vulgar 's.

Este florecimiento de tipos escriturarios se produce, como en el resto de Europa, también en la corona castellano-leonesa, donde tanto la instancia libraria como la documental ven multiplicarse en gran manera su producción.

En el campo de las escrituras librarias, es perfectamente aplicable a los manuscritos castellano-leoneses el esquema clasificatorio propuesto por G.I. Lieftinck, con sus cuatro categorías: textual, notular, cursiva y bastarda, y las subdivisiones según su tratamiento escriturario caligráfico (formata), común o corriente (currens) ${ }^{16}$, clasificación certeramente adecuada por A. Canellas en el caso que nos ocupa al introducir la subdivisión de estos tipos en dos subtipos, fracturada y redonda, que reflejan la realidad gráfica de los reinos de Castilla y León donde, tras unos inicios de trazado fracturado y anguloso, "típicamente» gótico, tanto en la escritura libraria como en la documental pasamos a trazados redondeados y continuos, más fáciles de ejecutar y que propician, en las escrituras cursivas, una mayor facilidad de establecer una escritura ligada, "ductus», que se obtiene mediante el retorno al uso de la pluma tallada centralmente ${ }^{17}$. Este hecho es perfectamente constatable en el campo de la escritura libraria a partir del siglo XIV con la aparición de la tradicionalmente denominada escritura semigótica o redonda ${ }^{18}$.

Hallamos, pues, en la corona castellano-leonesa constatación del uso de la escritura textualis formata, en sus versiones fracturada y redonda, fundamentalmente en los grandes corales y en libros de lujo. Textualis y Textualis currens, asimismo en ambas versiones, las vemos utilizadas tanto en códices universitarios ${ }^{19}$ como en códices diplomáticos, al igual que las bastardas.

Las cursivas irrumpen en el campo librario esencialmente de la mano de la literatura en lengua romance. De todos es conocida su utilización en la realización del manuscrito del «Libro del buen amor» ${ }^{20}$. También en códices

15 Cf. A. PetruCCI, Lezioni di storia della scrittura latina, "Corso istituzionale di Paleografia», Università degli Studi di Roma, Fac. di Lettere e Filosofia, anno acc. 1975-76, lez. XXV.

${ }_{16}$ Vid. G.I. LIEFTINCK, Pour une nomenclature de l'écriture livresque du période dite gothique. Essays s'applicant spécialement aux manuscrits originaires des Pays-Bas, en «Nomenclature des écritures livresques», pp. 13-34.

17 Cf. PetrucCI, Lezioni, lez. XXV.

${ }^{18}$ Cf. Millares, ob.cit., pp. 211-212.

19 Sobre manuscritos universitarios castellano-leoneses vid. G. BEAUjOIN, Manuscrits scientifiques médiévaux de l'Université de Salamanque et de ses "Colegios Mayores», Bordeaux, 1962.

${ }^{20}$ Cf. Canellas, ob.cit., lám. LIX y Millares, ob.cit., II, fig. 273. 
diplomáticos, de los que son claro exponente dos de los mandados confeccionar por el obispo de Oviedo D. Gutierre de Toledo, el «Libro de los Privilegios» y el "Libro Becerro" ". Se ejecuta, en estos casos, bien una cursiva con tendencia a la legibilidad por su mayor módulo y menores concesiones a las deformaciones de su morfología esencial, provocadas por las excesivas ligaduras, bien el tipo de cursiva formata, con una mayor tendencia a la caligrafización ${ }^{22}$. En cuanto al uso librario de las cursivas corrientes, creo más oportuno tenerlo en cuenta en el apartado de escrituras documentales, ya que los libros en los que se utiliza dentro de nuestra área son, por lo general, libros/documento: libros de cuentas, libros de actas y libros de copias diplomáticas, fundamentalmente registros.

En el campo documental predomina en el periodo gótico, como en el resto de los paises occidentales, el uso en la corona castellano-leonesa de la cursiva, fundamentalmente análoga en toda Europa, pero articulada en variedades nacionales, e incluso locales, y que es, como indica G. Cencetti, nominada en bloque por los paleógrafos como minúscula gótica notarial o cursiva góti$\mathrm{ca}^{23}$.

En el ámbito castellano-leonés se puede apreciar con toda nitidez una evolución clara de la cursiva gótica, desde una cursiva gótica inicial, propia del siglo XIII y primera mitad del XIV, de trazado anguloso y despiezado, "típicamente" gótica, que podemos, sin lugar a dudas, asimilar a la tradicionalmente denominada letra de albalaes, denominación notoriamente impropia, creada por el padre Terrenos y que, sin embargo, ha hecho fortuna entre los paleógrafos hispanos ${ }^{24}$. Esta cursiva gótica fracturada o angulosa da lugar a tres tipos diferentes, según la mayor o menor velocidad del «ductus» con el que se ha procedido a su ejecución.

Existe, pues, una cursiva gótica fracturada formata, utilizada fundamentalmente en la cancillería real en documentación no solemne emitida en pergamino, es decir, en las denominadas cartas abiertas ${ }^{25}$; es escritura de rasgos alargados, despiezada, de aspecto apretado y de fácil lectura. Es utilizada también por otras cancillerías y algunos notarios ${ }^{26}$.

Existe asimismo una cursiva gótica fracturada, de uso común, sin ningún tipo de concesiones a la estética, y que es utilizada tanto en la cancillería real

${ }^{21}$ Cf. F.J. FernÁNDez CONDE, Gutierre de Toledo, obispo de Oviedo (1377-1389). Oviedo 1978, pp. 278-279.

22 Cf. Millares, ob.cit., II, fig. 269. Aunque calificada como semigótica, el trazado de las formas alfabéticas nos sitúa ante una cursiva formata.

2 Cf. CENCETTI, ob.cit., p. 225.

24 Cf. Millares, ob.cit., II, figs. 193-194.

is Ibid., figs. 201, 204, 208 y 211.

${ }^{20}$ Cf. LuCAS, ob.cit., p. 63. 
para documentos menores en pergamino, compartiendo con la formata la redacción de cartas abiertas, como para documentos en papel, los mandatos. Es escritura muy ligada y por eso mismo con un frecuente desarrollo de ojillos en las astas ascendentes y descendentes de las formas alfabéticas ${ }^{27}$. Este tipo de escritura es la utilizada mayoritariamente por los notarios leoneses y escribanos públicos castellanos.

Existe, por último, una cursiva gótica fracturada currens o corriente, que se nos manifiesta fundamentalmente en las notas dorsales en los documentos notariales, en las rúbricas del personal de cancillería en los documentos emitidos por la misma y en anotaciones marginales de códices, ejecutadas con un trazado totalmente descuidado.

Pero a partir de la segunda mitad del siglo XIV se inicia en la escritura documental castellano-leonesa un proceso que A. Petrucci analiza en la escritura practicada en Italia y Francia a lo largo del siglo XIII ${ }^{28}$ y que consiste en la formación de un nuevo tipo de cursiva, caracterizada por su mayor número de ligaduras entre letra y letra, por el cuerpo redondeado de las formas alfabéticas y por el aspecto ornamental de los signos abreviativos, así como por lo poco acentuado de su claroscuro.

Es el momento en que el tipo redondo se hace patente en la cursiva documental castellano-leonesa, dando lugar a la gótica cursiva redonda, denominada tradicionalmente cortesana y que, tras un primer periodo de transición, al que suele calificarse de precortesana ${ }^{29}$, cristalizará también en tres subtipos: la gótica cursiva redonda formada o cortesana formata, trazada con mano no excesivamente rápida, y de la que hallamos ejemplos claros en la cancillería real castellana, en tiempos de los Trastámara ${ }^{30}$. Una gótica cursiva redonda o cortesana, utilizada comunmente tanto en cancillerías como en oficios notariales ". Y, por último, una gótica cursiva redonda corriente, que a finales del siglo $\mathrm{XV}$ desembocará en la denominada comunmente procesal $^{32}$, utilizada tanto en libros diplomáticos (libros de actas y acuerdos, registros, etc.) como en procesos judiciales.

Y es precisamente en el proceso de adaptación de la escritura gótica cursiva fracturada a la gótica cursiva redonda donde se van a producir los únicos cambios morfológicos en el alfabeto de la escritura gótica castellano-leonesa: la aparición de la tradicionalmente denominada sigma $(\sigma)$ y la a de lineta $(\alpha)$. En ambos casos se trata tan solamente de la adaptación gráfica de tres formas

27 Millares, ob.cit., II, figs. 205, 206, 209, 210 y 211.

${ }^{28}$ Cf. PetruCCI, Lezioni, lez. XXV.

29 Cf. Millares, ob.cit., I, p. 225.

310 Ibid., III, fig. 289.

"Ibid., figs. 288, 290, 291, 314 y 316.

i2 Ibid., figs. 315 y 321 . 
alfabéticas insolidarias en su morfología esencial ( $A, S$ y $Z$ ), en un sistema gráfico en el que prima la capacidad de establecer ligaduras tanto directas como inversas.

De hecho la sigma no es una forma esencialmente nueva, sino la convergencia o concurrencia en una misma forma de otras dos de distinto origen, cuya evolución puede seguirse con toda claridad en documentos de la primera mitad del siglo XIV, con el siguiente desarrollo:

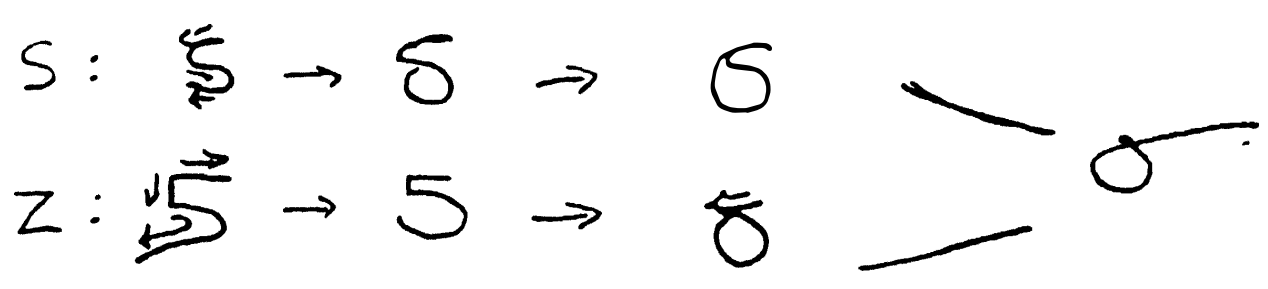

dando lugar a la duplicidad fonética para un mismo signo alfabético, duplicidad que ya manifestó, así como su probable origen, mediado el siglo XVI, Antonio de Torquemada ${ }^{33}$.

Significativamente es también el cambio de la a triangular, uncial, por la a de doble curva, semiuncial, a la que, en este caso, se cierra por su parte superior mediante una línea que determina su denominación (de lineta), por medio de la cual forma ligadura directa con la letra que la sigue, utilizándose el arranque de la primera de sus curvas-base para el enlace en ligadura inversa, quedando así convertida una forma alfabética plenamente insolidaria con una forma alfabética puente ${ }^{34}$.

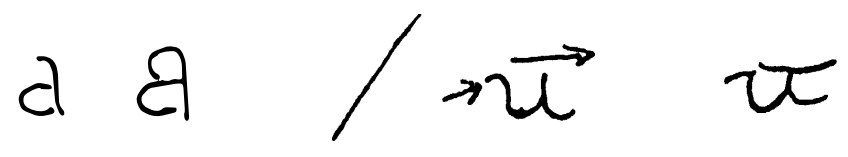

Pero en el campo documental vamos a encontrar también en Castilla y León utilizados tipos escriturarios básicamente librarios.

Tal es el caso de las textuales, tanto fracturada como redonda, que serán usadas frecuentemente en la expedición de los documentos más solemnes de la

${ }^{33}$ Cf. Manual de escribientes, Edic. de M.J. CANEllada de Zamora y A. ZamorA ViCENTE, Madrid, 1970 (Cit. Millares, ob.cit., I, p. 228).

${ }^{34}$ Sírvenos en este caso la a como indicativo de variante entre dos tipos de cursiva gótica, la fracturada y la redonda. Ya ha sido utilizada para tal fin en escritura libraria por W. OESER, Das 'a' als Gründlage für Schriftvarianten der gotischen Buchschrift, «Scriptorium», XXV-1 (1971). pp. 25-45. 
cancillería real castellano-leonesa, los privilegios rodados y las cartas plomadas de concesión o confirmación de privilegios ${ }^{33}$.

La textualis formata queda reducida en el ámbito documental, solamente con efectos decorativos, a las palabras iniciales de algunos documentos solemnes o a los «incipits» de códices diplomáticos ${ }^{36}$.

Tras este breve y sintético recorrido a través de la escritura gótica castellano-leonesa, creo quedan patentes las tareas a realizar en su campo por los estudiosos de la historia de la escritura.

Hay, en primer lugar, que tender a la adecuación de las anticuadas nomenclaturas al uso a los nuevos sistemas de denominación, tarea ya iniciada en otros países ${ }^{37}$ y no exenta de dificultades, como claramente ha expuesto $P$. Spunar ${ }^{38}$.

Carecemos, por otra parte, de estudios en profundidad de las escrituras góticas librarias, dificultades, al igual que en el caso de la escritura carolina, aumentadas por la inexistencia del Catálogo de códices datados ${ }^{39}$.

Por lo que se refiere a escrituras documentales, faltan estudios parciales serios sobre escrituras notariales, para las cuales, y en el ámbito estudiado, solamente contamos con el efectuado por M. Lucas sobre los notarios de Santiago de Compostela ${ }^{40}$. Igualmente carecemos de estudios sobre escrituras de oficios cancillerescos, a los que se han realizado aproximaciones en obras cuya finalidad esencial no era en absoluto el estudio pormenorizado de la escritura ${ }^{41}$. Del mismo modo, serían fructíferos estudios de ámbito regional, similares a los realizados por $W$. Heinemeyer con documentos pertenecientes a la zona del Rhin medio ${ }^{42}$, paralelo al realizado por J. Stiennon en la diócesis de Lieja en el tránsito del ciclo carolino al gótico ${ }^{43}$.

Todo ello serviría para sentar unas bases sólidas que, tras la realización de estudios comparativos, nos conducirían a la extracción de conclusiones válidas

"Cf. Millares, ob.cit., I, p. 195.

${ }^{36}$ Ibid., III, fig. 307.

3 Por lo que respecta a terminología de escrituras góticas, es de tener en cuenta el trabajo de M. SteInManN, Textualis formata, "Archiv für Diplomatik», XXV (1979). De significado más general vid. J.P., GUMBERT, A Proposal for a cartesian Nomenclature, en «Essays Presented to G.I.Lieftinck», 4, Amsterdam 1976, pp. 45-52.

${ }^{38}$ Paleographical Difficulties in Defining an individual Script, "Ibíd», págs. 62-68.

39 Como aportación al tema vid. J. TRENCHS OdenA, El Fuero de Úbeda. Estudio paleográfico, en «Fuero de Úbeda», Valencia, 1979, pp. 231-240.

${ }_{40}$ Vid. nota 1.

${ }^{41}$ Vid. M.L. PARDo RodríGUeZ, Huelva y Gibraleón. Documentos para su bistoria (1282-147.5), Huelva, 1980.

${ }^{42}$ Studien zur Geschichte der gotische Urkundenschrift, 2." ed., Köln-Wien, 1982.

${ }^{43}$ L'écriture diplomatique dans la diociese de Liège. 
para este ciclo escriturario significante de una época de gran relevancia sociocultural.

\title{
M. ${ }^{2}$ JOSEFA SANZ FUENTES Universidad de Oviedo
}

\begin{abstract}
RÉSUMÉ
L'histoire des écritures utilisées dans les règnes de la Couronne de Castille et Léon pendant le bas Moyen Age souffre, jusqu'à maintenant, d'un manque d'études minutieuses se rapportant à différents lieux, chronologies et usages et aussi d'études approfondies sur un ensemble clairement structuré. Cet article prétend, compte tenu du peu d'espace dont je disposais, faire ressortir les carences matérielles existantes sur le thème et ouvrir de nouvelles perspectives, basées fordamentalement sur la réalisation d'études partielles et sur l'adaptation de la terminologie applicable aux types authentiques utilisés pendant un si long laps de temps.
\end{abstract}

\section{SUMMARY}

The history of the scripts used in the reign of the Crown of Castilla and Leon during the early Middle Ages lacks, till now, of meticulous studies related to different places, to chronology and to uses of wide general studies clearly structured. This essay would like, in the reduced space I have been allowed to dispose of, to point out the material shortages about this topic and to open new horizons, mainly based on the realization of partial studies and on the adjustment to the applicable terminology of the genuine types used during a wide period of time. 